\title{
How human and nature shake hands: the role of no-conspiracy in physical theories
}

\author{
Gábor Hofer-Szabó*
}

\begin{abstract}
No-conspiracy is the requirement that measurement settings should be probabilistically independent of the elements of reality responsible for the measurement outcomes. In this paper we investigate what role no-conspiracy generally plays in a physical theory; how it influences the semantical role of the event types of the theory; and how it relates to such other concepts as separability, compatibility, causality, locality and contextuality.
\end{abstract}

Keywords: no-conspiracy, separability, compatibility, causality, locality, contextuality

\section{Introduction}

As the old bon mot has it, in experiment human and nature shake hands. This portrayal of the experiment as the celebration of a good business pact between two parties highlights two features of experimentation, namely that both human and nature are equally contributing to its success and that both parties are being independent. This independence is the topic of the present paper.

In the foundations of quantum mechanics probably the most significant research project has been for decades to precisely identify and conceptually analyze those assumptions that go into the derivation of the Bell inequalities and can be made responsible for their violation in the EPR scenario. Locality, factorization, Common Cause Principle, determinism-these were the main concepts and principles on the table. There was, however, one additional premise which, though being indispensable in the derivation of the Bell inequalities, remained much more obscure concerning its status, meaning and relation to the other premises.

The palpable evidence for this embarrassment around this assumption is that there has not even been coined a name for it. It has been referred to by many names such as (no) "conspiratorial entanglement" (Bell, 1981), "hidden autonomy" (Van Fraassen, 1982), "independence assumption" (Price 1996), "free will assumption" (Tumulka, 2007), "measurement independence" (Sanpedro, 2013), (no) "superdeterminism" (Price and Wharton, 2015), and-probably in its most wellknown form-"no-conspiracy" (Hofer-Szabó, Rédei and Szabó, 1999; Placek and Wroński, 2009). This latter is the phrase we are going to use in this paper.

The fact that no-conspiracy has been used by so many names attests that there is a wide range of topics which it can be related to. It has been explicitly addressed by Bell in his

${ }^{*}$ Research Center for the Humanities, Budapest, email: szabo.gabor@btk.mta.hu 
1981 paper and its rejection has been qualified as "even more mind boggling than one in which causal chains go faster than light" (Bell, 1981, p. 57). No-conspiracy made its way into the philosophy of physics via Van Fraassen's 1982 careful analysis of the assumptions leading to the Bell inequalities. Ever since these two influential papers no-conspiracy has been given some attention in the philosophy of science. A topic gaining probably the greatest philosophical interest was that how no-conspiracy is related to free will. The first to identify conspiracy as a lack of free will was Bell $(1977,1981)$ himself and has been followed by many others (Price 1996; Conway and Kochen, 2006; Tumulka, 2007, Price and Wharton, 2015).

The present paper does not concern any of the topics mentioned above: neither free will, nor EPR, nor Bell inequalities. It does not investigate no-conspiracy at the level of the specific scientific theories such as quantum mechanics, quantum field theory, etc. (For this see (Bell, 1977, 1981), (Butterfield, 1995), (Sanpedro, 2013, 2014), (Hofer-Szabó, Rédei and Szabó, 2013), (Price and Wharton, 2015)). Our aim is more general: to investigate what role no-conspiracy plays in a physical theory. To this aim in Section 2 we will first unfold a general scheme of the ontology of a physical theory. We will discern two event types making the ontology: measurement event types and elements of reality. Measurement event types can be of two types: measurement settings and measurement outcomes. We will clarify how measurement settings and measurement outcomes provide semantics for a physical theory. To illustrate the general scheme we introduce a toy model in Section 3 which will then be used throughout the paper. No-conspiracy enters in Section 4. Here we show how the presence of no-conspiracy can deprive measurement settings and measurement outcomes of their semantical role and directs them into pragmatics. In Section 5 some examples will be given for situations when no-conspiracy is violated. In Sections 6 to 10 we will investigate in turn the relationship of no-conspiracy to such concepts as separability, compatibility, causality, locality and contextuality. We conclude with a discussion in Section 11.

This paper is written in the down-to-earth physicalist philosophy of László E. Szabó to whom I dedicate it.

\section{The ontology of experiment}

In this Section we expose the main philosophical ideas lying behind our approach in a concise manner. In the following Section all these general considerations will be made concrete on a simple toy model. The approach we are following here is a strict actualist approach where the key concepts such as causality, probability, etc. all supervene on particulars instantiating certain event types in a Humean manner. This framework is certainly not necessary to address the question of no-conspiracy; I presume that most claims of the paper also hold in other metaphysical frameworks. I follow this approach simply because the present paper is part of a larger research project aiming to explore how far one can get in understanding physical theories and especially quantum mechanics within a Humean framework.

A physical theory can be reconstructed as a formal system plus a semantics connecting the formal system to the world. The formal system consists of a formal language with some logical axioms and derivation rules, some mathematical and physical axioms. The semantics provides an interpretation for the formalism; it connects the formal system to reality. Note that here 'semantics' does not mean a connection between the formal system and some models of the 
system as in model theory; here semantics means a down-to-earth physical interpretation of the formal system. We stress again that the semantics is an indispensable part of a physical theory. A formal system in itself is not yet a physical theory (Szabó, 2011).

The semantics settles the ontology of the theory. This can be done in many ways but typically the semantics fixes the ontological types or categories out there in the world and provides some means to decide when a certain token falls in the category of a given type making a certain sentence of the theory true. The types and tokens which we will be interested in here are event types and token events. The ontology of a physical theory is an event algebra constructed from these event types. Note that concerning the ontology of the types our approach is not committed metaphysically either to the realist nor to the nominalist camp.

Physical theories are verified by experiments. The rough picture of an experiment is the following. An experimenter performs a procedure by setting a measurement apparatus in a certain way, obtaining a measurement outcome and repeating this procedure many times. The two essential ontological categories of an experiment are the measurement settings and the measurement outcomes. These categories are event types just as the other ontological types of the theory. The token events are the instances of these event types in the different runs of the experiment. Sometimes I will simply refer to these token events as the runs of the experiment.

Measurement settings and measurement outcomes do not appear directly in the textbook form of a theory but they are indispensable part of the semantics (not of pragmatics!): without them the theory cannot be linked to reality. More than that, these two types are the only types an experimenter has direct empirical access to. Everything else posited by the theory has to ultimately boil down to some relations between these observable categories. To be more specific, any deductive or inductive relation between the ontological types of the theory has to be accounted for in terms of correlations between the token events falling in the category of measurement settings and measurement outcomes. As the empiricist thesis teaches, one has no other access to physical reality than via observation.

Correlations between measurement settings and measurement outcomes can be accounted for in terms of probabilities. In our actualist framework the probability of an outcome type is understood as the long-run relative frequency of those runs of the experiment which fall in that type if the experiment is repeated appropriately many times. Specifically, the probability of an outcome given a certain measurement setting is simply the number of those runs which fall in both the type of the outcome and the setting divided by the number of those runs which fall in the type of the setting. More importantly, any probability assignment to any ontological type to which we have no direct empirical access must be based on type assignments to the individual runs of the experiment in the long-run frequency sense: the probability of a given type is $p$ only if the relative frequency of the individual runs (instances) falling in the type in question is $p$. Probability supervenes on the Humean mosaic of token events.

In order to account for the observable measurement outcomes physical theories typically introduce a further, not directly accessible event type, which we will call elements of reality. In this sense our approach is scientifically realist. Elements of reality come in two sorts: they can either determine the measurement outcomes for a given measurement setting for sure, or they can fix only the probability of the measurement outcomes. We will call the first event type property and the second event type propensity. Whereas measurement outcomes are clearly causally influenced by and therefore probabilistically dependent on the elements of reality, it is 
not a priori clear what the relation between the measurement settings and the elements of reality should be. This is what we are going to analyze in what comes.

\section{A toy model}

Let us make these abstract considerations more concrete on a simple model. (For a general scheme of a physical theory see the Appendix.) Consider a box containing colored dice (Szabó, 2008). Let us try to develop a physical theory of this system. Whatever theory we develop, the semantics of the theory has to minimally specify the measurement settings and measurement outcomes. These are the categories which are directly accessible for an experimenter. Suppose that the measurement settings are the following:

$a_{1}$ : drawing a die from the box and checking its color

$a_{2}$ : drawing a die from the box, throwing it and checking the number on its upper face

Suppose furthermore that the measurement outcomes are

$A_{1}^{i}$ : the color of the die is black $\left(A_{1}^{1}\right)$ or white $\left(A_{1}^{2}\right)$

$A_{2}^{j}$ : the number on the upper face of the die is $j(j=1 \ldots 6)$

So the semantics of the theory posits the following event types: the measurement settings $a$ with two subcategories $a_{1}$ and $a_{2}$, and the measurement outcomes $A$ with two plus six subsubcategories $A_{1}^{i}$ and $A_{2}^{j}$.

As the experimenter is repeating the experiment, the token events, that is the runs falling in the different event types, are accumulating giving rise to a probabilistic description of the experiment. She can calculate for example the conditional probability of obtaining a black die on the condition that she had performed the color measurement:

$$
p\left(A_{1}^{1} \mid a_{1}\right)=\frac{\#\left(A_{1}^{1} \wedge a_{1}\right)}{\#\left(a_{1}\right)}
$$

This probability is empirically accessible: one just reads off from the relative frequency of the measurement outcomes and measurement settings. (Here we set aside problems concerning the convergence of the relative frequencies.)

The experimenter can of course try to enrich her theory and introduce a new ontological category into her theory. The motivation behind this move is to obtain an answer to the question: "Why was the outcome of the color measurement black in a certain run of the experiment?" A natural answer to this question is to say: "Because the die itself was black." This answer amounts to introducing a third event type into our ontology, which we will call property. What is a property?

The defining feature of the property black is the following: whenever a die with the property black is subjected to a color measurement, the outcome will always be black. Denote the property black by $\alpha_{1}^{1}$ and the property white by $\alpha_{1}^{2}$. (So our notation is the following: we use lower case Latin letter for the measurement settings $(a)$; capital Latin letters for the measurement outcomes $(A)$; and Greek letters for the elements of reality $(\alpha)$.) The property black is an event type and each token event that is each run of the experiment can be characterized by either falling into 
this event type or not. Therefore, one can also meaningfully speak about the probability of the property black, $p\left(\alpha_{1}^{1}\right)$, as the long-run relative frequency of those runs of the experiment which fall into the event type $\alpha_{1}^{1}$. Consequently, one can also express the defining feature of the property black and white in terms of probabilities as follows:

$$
p\left(A_{1}^{i} \mid a_{1} \wedge \alpha_{1}^{k}\right)=\delta_{i k} \quad i, k=1,2
$$

That is in each run of the experiment when the die was black and the color has been measured, the outcome was black and never white; and in each run of the experiment when the die was white and the color has been measured, the outcome was white and never black. A property is nothing but an event type which, if instantiated and measured in a certain run of experiment, brings with it a definite outcome.

Let us now go over to the case of throwing the dice and ask a similar question to that of the color measurement: "Why does the outcome six come up with a certain probability in the experiment?" Here the natural answer is this: "Because the die has a certain mass distribution." This leads us to introducing another event type which we will call propensity.

Suppose that the box is containing dice with two different mass distributions. Denote them by $\alpha_{2}^{1}$ and $\alpha_{2}^{2}$. Here the lower index 2 indicates that the measurement setting is of the second type, namely checking the upper face of the die (and not the color), and the upper index discerns the two mass distributions. The mass distribution $\alpha_{2}^{1}$ is again an event type just as $\alpha_{1}^{1}$, the property black was. In every single run of the experiment it is either instantiated or not that is each die has either the mass distribution $\alpha_{2}^{1}$ or not. Hence one can speak about the probability $p\left(\alpha_{2}^{1}\right)$ as the relative frequency of those runs which fall into the event type $\alpha_{2}^{1}$. If a die with mass distribution $\alpha_{2}^{1}$ is drawn from the box and thrown, then let the probability of its coming up $j$ be denoted by $q^{j 1}$. Similarly, if a die with mass distribution $\alpha_{2}^{2}$ is drawn from the box and thrown, then the probability of coming up $j$ is $q^{j 2}$. This means that the mass distribution of a given die fixes the probability of the die coming up with a certain face upon throwing. In terms of probabilities this can be expressed as follows:

$$
p\left(A_{2}^{j} \mid a_{2} \wedge \alpha_{2}^{l}\right)=q^{j l} \quad j=1 \ldots 6, l=1,2
$$

where $\sum_{j} q^{j l}=1$ for $l=1,2$.

Metaphysically, the new event type $\alpha_{2}$ is the propensity of the die to come up with a certain face in the second type of measurement setting. Note that the propensity here is not something which the notion of probability should be reduced to as in the literature on the interpretations of probability. Here propensity is an event type and probability is simply long-run relative frequency. Moreover, one can meaningfully speak about the "probability of a given propensity" as the long-run frequency of those token events which instantiate the event type of the propensity in question.

Also observe that a property mathematically differs from a propensity only in that the $q^{j l}-\mathrm{s}$ fixing the conditional probabilities are all either 0 or 1 for the properties, whereas they can be any number between 0 and 1 for the propensities. Being black fixes the measurement outcomes for the color measurement, whereas having mass distribution $\alpha_{2}^{1}$ fixes only the probability of obtaining a six. The defining equation (1) of properties is a special case of the defining equation (2) of propensities. Still, it is worth discerning these two event types. If in a given theory 
the probabilities, correlations, etc. of the measurement outcomes can all be accounted for by postulating purely properties then the theory can rightly be called deterministic, whereas if propensities are also needed then the theory is indeterministic.

To sum up, in our "theory of dice" we have two measurement event types, the event type of measurement settings and the event type of measurement outcomes. Beyond these we can introduce into our ontology two elements of reality for explanatory purposes, the event type of properties, $\alpha_{1}$, with two subcategories $\alpha_{1}^{1}$ (black) and $\alpha_{1}^{2}$ (white); and the event type of propensities, $\alpha_{2}$, with two subcategories $\alpha_{2}^{1}$ (first mass distribution) and $\alpha_{2}^{2}$ (second mass distribution). From now on we will coin the term measurement event type for measurement settings and measurement outcomes and element of reality for properties and propensities. The event algebra of the theory will be composed as the Boolean combination of the measurement event types and elements of reality. This algebra will be built up from $2 \cdot(2 \cdot 6) \cdot(2 \cdot 2)$ atomic events associated to the different combinations of measurement settings, measurement outcomes, properties and propensities. Each run of the experiment will instantiate an element of this algebra. Probabilities enter the theory by simply counting how many runs are instantiating certain elements of the algebra.

\section{No-conspiracy}

So far, so good. But physics is a procedure to move from the observable to the unobservable. Do we have any means to infer from the first two event types to the second two? Can we say something about properties and propensities based on measurement settings and measurement outcomes?

Here is a sufficient condition which entitles us to such an inference. Suppose that the elements of reality are probabilistically independent of the measurement settings. In case of the properties this means that

$$
p\left(a_{1} \wedge \alpha_{1}^{k}\right)=p\left(a_{1}\right) p\left(\alpha_{1}^{k}\right) \quad k=1,2
$$

in case of the propensities:

$$
p\left(a_{2} \wedge \alpha_{2}^{l}\right)=p\left(a_{2}\right) p\left(\alpha_{2}^{l}\right) \quad l=1,2
$$

Taking the conjunctions we obtain:

$$
p\left(a_{1} \wedge a_{2} \wedge \alpha_{1}^{k} \wedge \alpha_{2}^{l}\right)=p\left(a_{1} \wedge a_{2}\right) p\left(\alpha_{1}^{k} \wedge \alpha_{2}^{l}\right) \quad k, l=1,2
$$

Now, consider all those other equations which arise from (5) by substituting one or more event types by their complements; for example:

$$
p\left(\sim a_{1} \wedge a_{2} \wedge \alpha_{1}^{k} \wedge \sim \alpha_{2}^{l}\right)=p\left(\sim a_{1} \wedge a_{2}\right) p\left(\alpha_{1}^{k} \wedge \sim \alpha_{2}^{l}\right) \quad k, l=1,2
$$

Including (5) one obtains thus altogether $2 \cdot 2 \cdot 4 \cdot 4=64$ equations. Let us refer to this set of 64 equations as no-conspiracy. No-conspiracy expresses a probabilistic independence between the various Boolean combinations of measurement settings and the various Boolean combinations of 
elements of reality. To make reference easier we will sometimes refer solely to (5) as no-conspiracy requirement without mentioning the other 63 equations arising from complementation.

No-conspiracy does us a great service: we can reproduce the observable probabilities of the theory in terms of the probabilities of the elements of reality. For example the conditional probability $p\left(A_{1}^{1} \mid a_{1}\right)$ of obtaining a black die upon color measurement turns out to be just the probability $p\left(\alpha_{1}^{1}\right)$ of the property black:

$$
\begin{aligned}
p\left(A_{1}^{1} \mid a_{1}\right) & =\frac{p\left(A_{1}^{1} \wedge a_{1}\right)}{p\left(a_{1}\right)}=\frac{\sum_{k} p\left(A_{1}^{1} \wedge a_{1} \wedge \alpha_{1}^{k}\right)}{p\left(a_{1}\right)}=\frac{\sum_{k} p\left(A_{1}^{1} \mid a_{1} \wedge \alpha_{1}^{k}\right) p\left(a_{1} \wedge \alpha_{1}^{k}\right)}{p\left(a_{1}\right)} \\
& =\frac{\sum_{k} p\left(A_{1}^{1} \mid a_{1} \wedge \alpha_{1}^{k}\right) p\left(a_{1}\right) p\left(\alpha_{1}^{k}\right)}{p\left(a_{1}\right)}=\sum_{k} p\left(A_{1}^{1} \mid a_{1} \wedge \alpha_{1}^{k}\right) p\left(\alpha_{1}^{k}\right) \\
& =\sum_{k} \delta_{1 k} p\left(\alpha_{1}^{k}\right)=p\left(\alpha_{1}^{1}\right)
\end{aligned}
$$

where we used only the theorem of total probability, the defining feature (1) of a property and no-conspiracy (3).

By similar reasoning we can reproduce the conditional probability $p\left(A_{2}^{6} \mid a_{2}\right)$ of obtaining the outcome six upon "upper face" measurement in terms of weighted averages of the probability of propensities $p\left(\alpha_{2}^{l}\right)$ :

$$
p\left(A_{2}^{6} \mid a_{2}\right)=q^{61} p\left(\alpha_{2}^{1}\right)+q^{62} p\left(\alpha_{2}^{2}\right)
$$

Equations (7) and (8) are of central importance. They explain why in the text book form of a physical theory one need not speak about measurement settings and measurement outcomes. If no-conspiracy holds, then the conditional probabilities of the measurement outcomes on measurement settings simply mirror the (unconditional) probabilities of the elements of reality (properties and propensities). Consequently, the deductive and inductive relations between the measurement event types simply reveal deductive and inductive relations between the elements of reality. For example, observing the relation that the probability of a die coming up six is higher than that of being black

$$
p\left(A_{2}^{6} \mid a_{2}\right)>p\left(A_{1}^{1} \mid a_{1}\right)
$$

reveals the unobservable fact that

$$
q^{61} p\left(\alpha_{2}^{1}\right)+q^{62} p\left(\alpha_{2}^{2}\right)>p\left(\alpha_{1}^{1}\right)
$$

More than that, the relations between measurement settings and measurement outcomes do not just reveal the hidden relations between the unobservable categories but by the same move they also seem to make measurement event types superfluous. If the role of these "surface" relations is simply to reflect the deep structural relationships of the unobservable categories with which real physics is concerned - then why one would care about them? Why one would care about measurement settings and measurement outcomes if one can also speak about the "real stuff" directly? In short, no-conspiracy can contribute to delegating measurement settings and measurement outcomes from semantics to mere pragmatics. 
May this rationale be as fruitful in displaying textbook theories as it is, in a philosophical reflection, I think, one should not concede that no-conspiracy blurs the general semantical role of measurement settings and measurement outcomes. Just recall the general frame: a physical theory is a formal system plus a semantics connecting the formal system to the world. The very two categories which lend empirical meaning to a physical theory are the measurement settings and the measurement outcomes. They are the only event types which an observer have direct access to. Consequently, they cannot be eliminated from a physical theory - neither by appealing to no-conspiracy, nor by appealing to anything else. Otherwise the whole theory would lose its empirical content. It would turn into an uninterpreted formalism. No consideration can deprive a physical theory of those constituents which make up its semantics.

But let us return now to no-conspiracy. What if no-conspiracy does not hold? In this case the inference from the measurement event types to the elements of reality via (7) and (8) is not possible. But does it make the knowledge of the unobservable categories impossible? Is no-conspiracy a kind of Kantian "condition of the possibility of experience"?

Some seem to think so. In his famous 'cat' paper Schrödinger (1935) likens the free measurement choice of the EPR experiment to a situation when a class of students are asked a set of question such that each student may be asked any of questions. If the answer to the questions are all correct, then one can conclude that all students know all answers. Analyzing Schrödinger's example Maudlin (2014) writes the following:

"Recall Schrödinger's class of identically prepared students. We are told they can all answer any of a set of questions correctly, but each can only answer one, and then forgets the answers to the rest. It's an odd idea, but we can still test it: we ask the questions at random, and find that we always get the right answer. Of course it is possible that each student only knows the answer to one question, which always happens to be the very one we ask! But that would require a massive coincidence, on a scale that would undercut the whole scientific method." (Maudlin, 2014 p. 23)

In short, the independence of the measurement choices and the elements of reality is a precondition of pursuing science per se. But is it really so?

\section{When no-conspiracy does not hold}

Consider the following examples:

Example 1. Suppose that the black painting on the dice is not durable enough: if you just touch the dice, the black color is wearing off it and it turns white.

Example 2. Suppose that each die is filled with a high viscosity fluid which can stream and swirl inside the die. By every throw the fluid is put in motion which changes the mass distribution of the die and hence the propensity of the outcome at that very throw.

Example 3 is special case of Example 2. Suppose again that the dice are filled with a fluid which can stream inside them before tossing. But by tossing the dice (due to the heavy shaking, say) the fluid "freezes out" in such a biased way that the dice can come up with only one definite face. 
The above three examples are all illustrating a situation when no-conspiracy is violated. In the first example the property $\alpha_{1}^{1}$ (black) has turned into another property $\alpha_{1}^{2}$ (white) as a result of the measurement setting $a_{1}$ (drawing a die from the box). In the second example the propensity $\alpha_{2}^{1}$ (first mass distribution) has turned into another propensity $\alpha_{2}^{2}$ (second mass distribution) as a result of the measurement setting $a_{2}$ (tossing a die). Finally, in the third example we find a change of category. Recall that properties and propensities differed only in whether they determined the outcome for sure or only up to a certain probability. In the third example there was some non-trivial probability for the different faces of the dice to come up before the throw. After the tossing, however, the die could come up only with a given face. That means that here a propensity (one sort of mass distribution) has been turned into a property (a special mass distribution exactly fixing the outcome) as a result of the measurement setting $a_{2}$ (tossing a die). In each case no-conspiracy is violated. (For the relevance of these examples to the interpretations of quantum mechanics see (Gömöri and Hofer-Szabó, 2016).)

In the above three examples no-conspiracy was violated due to the causal influence of the measurement settings on the elements of reality. But it can also fail due to an opposite causal connection when the elements of reality have causal influence on the measurement settings:

Example 4. Suppose that touching the dice of the second mass distribution is unpleasant for your hand; so you toss them hastily rather then keep them in hand and check the color.

Yet another example for the violation of no-conspiracy is a common causal connection between the elements of reality and the measurement settings. It is a combination of example 1 and 4 .

Example 5. Suppose that the dice of the second mass distribution are too heavy to be tossed; so you rather perform a color measurement on them. Suppose furthermore that being heavy and having a second mass distribution have a common cause - say, these dice are being made in the same factory.

In all the above examples no-conspiracy was violated due to a causal connection between the measurement settings and the elements of reality. But is causal connection the only way to violate no-conspiracy? We come back to this question in Section 8.

Now, we go over to our central question: Under what circumstances can we adopt noconspiracy in our physical theory, and when are we forced to abandon it? In the upcoming five Sections we investigate five concepts in turn which can qualify the decision. They are separability, compatibility, causality, locality and contextuality.

\section{Separability}

Niels Bohr's notorious insistence on the use of classical concepts in the description of quantum phenomena is one of the hallmarks of his philosophy. In his contribution to the 1949 Einstein Festschrift Bohr writes:

It is decisive to recognize that, however far the phenomena transcend the scope of classical physical explanation, the account of all evidence must be expressed in classical terms. The argument is simply that by the word "experiment" we refer to a situation where we can tell others what we have done and what we have learned and 
that, therefore, the account of the experimental arrangement and of the results of the observations must be expressed in unambiguous language with suitable application of the terminology of classical physics. (Bohr 1949, p. 209).

Many Bohr scholars have made significant efforts to understand the meaning and role of Bohr's doctrine on the primacy of classical concepts. Camilleri and Schlosshauer (2015) argue that Bohr's doctrine is primarily a general epistemological thesis articulating the epistemology of experiment rather than a special interpretation of quantum mechanics (for this see also (Zinkernagel, 2015)). The epistemological problem according to Bohr is that whereas the very notion of experiment presupposes that the measured objects possess a definite state which is independent from the state of the measurement apparatus, quantum mechanics makes this distinction between object and apparatus ambiguous by treating the two as a single, composite, entangled system:

... the impossibility of subdividing the individual quantum effects and of separating the behaviour of the objects from their interaction with the measuring instruments serving to define the conditions under which the phenomena appear implies an ambiguity in assigning conventional attributes to atomic objects which calls for a reconsideration of our attitude towards the problem of physical explanation. (Bohr 1948, p. 317).

If entanglement between object and apparatus is the obstacle to an unambiguous description of quantum phenomena, then such a description in classical terms can be realized when the subsystems are not entangled, that is when they are separable. This is exactly Don Howard's (1994) suggestion for the reconstruction of Bohr's doctrine on classical concepts:

... for Bohr, classical concepts are necessary because they embody the assumption of instrument-object separability, and that such separability must be assumed, in spite of its denial by quantum mechanics, in order to secure an unambiguous and thus objective description of quantum phenomena. (Howard 1994, p. 209).

Howard's suggestion to analyze classical description in terms of separability boils down to the requirement to reproduce the statistical predictions of a given quantum phenomenon in terms of an "appropriate mixture." The state of a composite system is called separable, if it is a mixture that is a convex sum of product states of the components. Since product states represent probabilistically independent components, a mixture is simply a convex combination of these states which expresses a classical probabilistic correlation between the components. Mixtures give rise to a classical, ignorance interpretation of the statistics of the phenomenon under investigation. This analysis via the notion of an "appropriate mixture" has been picked up for example by Halvorson and Clifton (2002) who provide an elegant analysis of the EPR experiment from Bohr's perspective along the lines suggested by Howard.

But how separability as a reconstruction of Bohr's demand on classicality relates to noconspiracy as a kind of independence principle between measurement settings and the elements of reality attributed to the system? Clearly, separability is a broader concept than no-conspiracy: separability simply requires that the relation between the measurement settings and elements of reality should be expressed as a mixture of probabilistic independences; whereas no-conspiracy 
requires that the two should be probabilistically independent. In our toy model for example separability requires the probability of the color measuring and the system's possessing the property black to be the following:

$$
p\left(a_{1} \wedge \alpha_{1}^{1}\right)=\lambda_{1} p\left(a_{1}\right) p\left(\alpha_{1}^{1}\right)+\lambda_{2} p\left(a_{1}\right) p\left(\sim \alpha_{1}^{1}\right)+\lambda_{3} p\left(\sim a_{1}\right) p\left(\alpha_{1}^{1}\right)+\lambda_{4} p\left(\sim a_{1}\right) p\left(\sim \alpha_{1}^{1}\right)
$$

with any $\lambda_{i} \in[0,1]$ and $\sum_{i=1}^{4} \lambda_{i}=1$; whereas no-conspiracy requires that

$$
p\left(a_{1} \wedge \alpha_{1}^{1}\right)=p\left(a_{1}\right) p\left(\alpha_{1}^{1}\right)
$$

Observe that separability (11) does not give any restriction in our case; it simply means that $p$ is a classical probability which we already knew since we took probabilities to be relative frequencies.

All the five examples in the previous Section, though violating no-conspiracy, are completely classical; they provide an unambiguous description of how the unobservable properties or propensities change upon throwing the dice. They even provide a mechanism for the causal dependence. In Example 1 for instance when upon drawing the black color is wearing off the dice, obviously

$$
p\left(a_{2} \wedge \alpha_{1}^{1}\right) \neq p\left(a_{2}\right) p\left(\alpha_{1}^{1}\right)
$$

Throwing the dice and being black will not be probabilistically independent due to the causal relation between the two event types.

Thus, the "unambiguous language" requires only to attribute some properties to the system which stand in some classical probabilistic relation to the measurement settings but it does not require them to be probabilistically independent of one another. Hence, separability as a weaker requirement than no-conspiracy cannot be used to back the latter. (In addition, according to Howard even the demand on classicality as separability is too restrictive from perspective of a general epistemology of experiment.)

\section{Compatibility}

Now, let us go over to our second concept which is compatibility of the measurement settings. Up to now we have considered measurement settings only separately. Let us see now what happens when we perform a joint measurement.

Again, consider our toy model and suppose that we perform the measurement $a_{1} \wedge a_{2}$ that is we are drawing a die from the box, throwing it and checking its color and also the number on its upper face. Suppose that after performing both measurements we disregard the upper face and consider only the color. Suppose that we observe that the probability of the outcome black in this joint measurement is not the same as in the measurement $a_{1}$. That is we find that

$$
p\left(A_{1}^{1} \mid a_{1} \wedge a_{2}\right) \neq p\left(A_{1}^{1} \mid a_{1}\right)
$$

Let us call (14) incompatibility of the two measurements. Note that incompatibility does not mean here that $a_{1} \wedge a_{2}$ cannot be performed; it means that $a_{1}$ and $a_{2}$ are disturbing one another.

What is incompatibility a sign of? 
First, observe that the condition $a_{1}$ on the right hand side of (14) does not mean that we performed only $a_{1}$-this would be $a_{1} \wedge \sim a_{2}$. The condition $a_{1}$ means that we consider all the runs in which $a_{1}$ has been performed, irrespectively whether $a_{2}$ has been performed or not - that is $a_{1}=\left(a_{1} \wedge a_{2}\right) \vee\left(a_{1} \wedge \sim a_{2}\right)$. So what (14) expresses is that whether we perform $a_{2}$ or not does count in measuring $a_{1}$ and producing outcome $A_{1}^{1}$.

Generally, one can take two positions towards incompatibility. I will call the first the purist or Bridgmanian strategy and the second the stubborn strategy.

According to the purist strategy if the probability of the outcome of a given measurement can vary depending whether another measurement is performed or not, then this measurement is not yet well defined. Consider the following example. In a regiment two tests are performed: it is tested how good shots are the soldiers $\left(a_{1}\right)$ and how much alcohol they can drink $\left(a_{2}\right)$. Obviously, whether the second test is performed or not, crucially influences the outcome of the first. So the two tests are incompatible in the above sense (and not in the sense that they cannot be performed at the same time: they can-although it is not recommended). So the correct definition of the first test is this: let the soldiers shoot but do not give them alcohol $\left(a_{1} \wedge \sim a_{2}\right)$.

So the purist attitude towards (14) is that $a_{1}$ in itself is not yet a well defined measurement procedure since the probability of the outcomes depends on whether $a_{2}$ is performed or not. So instead of taking two measurement settings, $a_{1}$ and $a_{2}$, we should rather take four, $a_{1} \wedge a_{2}$, $a_{1} \wedge \sim a_{2}, \sim a_{1} \wedge a_{2}$ and $\sim a_{1} \wedge \sim a_{2}$ (in this latter case we do nothing). By this move we can eliminate incompatibility since the four new measurements are logically mutually exclusive. They cannot be co-performed and hence cannot disturb one another. Generally, the purist strategy is to take the conjunctions of incompatible measurements until they become either compatible or logically exclusive.

We call this strategy Bridgmanian since it is in tune with Bridgman's ideas on the correct definition of measurement unfolded for example in The Logic of Modern Physics:

Implied in this recognition of the possibility of new experience beyond our present range, is the recognition that no element of a physical situation, no matter how apparently irrelevant or trivial, may be dismissed as without effect on the final result until proved to be without effect by actual experiment. (Bridgman 1958, p. 3)

Returning to no-conspiracy, the Bridgmanian strategy renders all co-measurable measurements compatible with one another. Therefore, the problem of incompatibility disappears and we are back to our single case measurement scenario. The purist strategy teaches nothing new about no-conspiracy.

Let us go over to the stubborn strategy. I call it stubborn since it takes $a_{1}$ and $a_{2}$ to be correct measurement settings in spite of their incompatibility (14)? What does then (14) say about no-conspiracy?

This is a point where we need to go one step further concerning the relation between measurement event types and elements of reality. We need to specify how the elements of reality behave when jointly measured. Therefore suppose that the following relation also holds (in addition to (1) and (2)):

$$
p\left(A_{1}^{i} \wedge A_{2}^{j} \mid a_{1} \wedge a_{2} \wedge \alpha_{1}^{k} \wedge \alpha_{2}^{l}\right)=\delta_{i k} q^{j l} \quad i, k, l=1,2 ; j=1 \ldots 6
$$


Requirement (15) expresses a kind of non-disturbance relation between the measurements which can be better seen if we sum up first for $i$ then for $j$ :

$$
\begin{aligned}
& p\left(A_{1}^{i} \mid a_{1} \wedge a_{2} \wedge \alpha_{1}^{k} \wedge \alpha_{2}^{l}\right)=\delta_{i k}=p\left(A_{1}^{i} \mid a_{1} \wedge \alpha_{1}^{k}\right) \\
& p\left(A_{2}^{j} \mid a_{1} \wedge a_{2} \wedge \alpha_{1}^{k} \wedge \alpha_{2}^{l}\right)=q^{j l}=p\left(A_{2}^{j} \mid a_{2} \wedge \alpha_{2}^{l}\right)
\end{aligned}
$$

(Here the second equation in both rows are due to the defining equation (1) of the property and (2) of the propensity, respectively.) (16) and (17) express that the probability of an outcome conditioned on an element of reality and a measurement setting does not change by further conditioning it on other elements of reality or measurement settings. From (16) (where the element of reality is a property) it also follows that

$$
p\left(A_{1}^{i} \mid a_{1} \wedge a_{2} \wedge \alpha_{1}^{k}\right)=p\left(A_{1}^{i} \mid a_{1} \wedge \alpha_{1}^{k} \wedge \alpha_{2}^{l}\right)=p\left(A_{1}^{i} \mid a_{1} \wedge \alpha_{1}^{k}\right)
$$

Now, suppose that no-conspiracy also holds that is

$$
p\left(a_{1} \wedge a_{2} \wedge \alpha_{1}^{k} \wedge \alpha_{2}^{l}\right)=p\left(a_{1} \wedge a_{2}\right) p\left(\alpha_{1}^{k} \wedge \alpha_{2}^{l}\right) \quad k, l=1,2
$$

From (15) and (19) it is easy to show (via a derivation similar to (7)) that

$$
p\left(A_{1}^{1} \mid a_{1} \wedge a_{2}\right)=p\left(A_{1}^{1} \mid a_{1}\right)
$$

in contradiction to incompatibility (14). This means that incompatibility between the measurements implies that we have to abandon either the non-disturbance of the measurement procedures (15) or no-conspiracy (19).

Thus, in case of the stubborn strategy compatibility of the measurement settings is a good sign of that both non-disturbance and no-conspiracy hold; and incompatibility is a good sign of that either the one or the other is violated. Whether to blame the one or the other is a question for further investigation.

\section{Causality}

Our third concept in the row is causality. In Section 5 we saw several examples for causal connections between the measurement settings and the elements of reality. In Example 1 for instance we supposed that the black painting on the dice is not durable enough and if one touches the dice, the color black is wearing off. Causal connection between elements of reality and measurement settings is a prime source of no-conspiracy.

Causal connection comes in two sorts. It can be either a direct causal connection as in Examples 1 to 4; or it can be a common causal connection as in Example 5. Reichenbach's Common Cause Principle states that all correlations should be accounted for by one of the two causal connections. On the other hand, probabilistic independence between the measurement settings and the elements of reality is a sign of causal independence (assuming that causal effects do not cancel one another). Hence, no-conspiracy can be ensured if any causal connection between the measurement settings and the elements of reality can be excluded.

Before turning to this point, first we need to clarify what we mean by a causal connection between two event types, say, the color measurement, $a_{1}$, and the property black, $\alpha_{1}^{1}$. By that 
we mean that the color measurement and the property black are causally related in a tokenwise manner. In other words, there is a pairing of token events instantiating these two types such that for each pair the token events of the pair stand in either a direct or a common causal connection to one another. But how to create pairs?

Consider a certain run of the experiment which instantiates $a_{1} \wedge \alpha_{1}^{1}$. Up to now we treated this run of the experiment as one single run in which one performed a color measurement and the property of the dice which has been drawn was black. How can the color measurement cause the property black in this single run? If this run of the experiment is taken as one single token event, then there can be no tokenwise causal connection; simply because we have only one token. In order to have a causal connection, one needs to decompose this one single run of the experiment instantiating $a_{1} \wedge \alpha_{1}^{1}$ into a pair of token events such that the one token event instantiates $a_{1}$ and the other token event instantiates $\alpha_{1}^{1}$. In order to speak about a tokenwise causal relation, one token event is not enough. One possibility to perform this decomposition is to say that the first token event occurred here and the other token event occurred over there. Localization is a typical method for individuation. We come back to the question of localization in the next Section.

Now, suppose that we can separately individuate the token events of the color measurement and the token events of the property black. Then a causal connection between $a_{1}$ and $\alpha_{1}^{1}$ means that for each pair either the token instantiating $a_{1}$ is the cause of the token instantiating $\alpha_{1}^{1}$; or vice versa; or there is a third token instantiating a third event type which is the common cause of both. Is there a way to exclude both a direct and also a common causal connection between the token events and by this to ensure no-conspiracy? What might come to mind first is to rely on some locality consideration. This is the topic of the next Section.

\section{Locality}

Is there a spatiotemporal arrangement of the event types $a_{1}$ and $\alpha_{1}^{1}$ such that one can safely say that all possible causal connections between the measurement settings and the elements of reality are shielded off? Suppose that we take a snapshot of the world and it turns out that the pairs of token events instantiating the color measurement and the property black are localized in spacelike separated regions. Thus, in the first run of the experiment the token event instantiating $a_{1}$ is spacelike separated from the token event instantiating $\alpha_{1}^{1}$; and similarly for the second, third, etc. run. This is the best scenario a spacetime localization can provide for causal independence. Does it guarantee that there is no causal and hence probabilistic dependence between $a_{1}$ and $\alpha_{1}^{1}$ ? As one expects, the answer to this question is no.

Even if the token events of each pair are spacelike separated, they can still be causally related to one another both in a direct and also in a common causal way. As for direct causal connection, just note that in order to produce a measurement outcome these two token events need to interact somewhere in spacetime. Hence even if they are spacelike separated at a certain moment, they will not be so at the moment of bringing about the outcome black. Therefore their direct causal effect on one another at the time of their interaction cannot be excluded based on the fact that at a previous time they were localized in a spacelike separated way. The situation is similar or even worse in case of a common cause. Even if the two token events are spacelike separated, 
there well can be a common cause in their common past causally influencing both.

To sum up, locality considerations do not help us in excluding causal connections and hence to ensure no-conspiracy. Thus, we have fallen back to the situation in the previous Section: to guarantee no-conspiracy we need to exclude causal connection in some way without making use of spatiotemporal considerations.

\section{Contextuality}

Up to now it may have appeared that the only source for the violation of no-conspiracy is a causal connection between the elements of reality and the measurement settings. However, there is a further way to violate no-conspiracy which is not related to causality. Two events can be correlated even if they are not causally related; namely if they logically depend on one another. This leads us to the problem of contextuality.

A little reflection on the definition of property and propensity can convince us that (1) and (2) say nothing about whether the elements of reality and the measurement settings are logically independent or not. It can well be the case that by specifying the measurement setting we partly specify also the elements of reality. Consider the following example:

Example 6. Let $\alpha_{2}^{1}(x)$ denote the following property of the dice: the mass distribution of the dice is of the first type and the initial conditions (position plus momentum) of its toss is $x . \alpha_{2}^{1}(x)$ is obviously a property since together with the toss $a_{2}$ it determines the upper face for sure; that is

$$
p\left(A_{2}^{j} \mid a_{2} \wedge \alpha_{2}^{1}(x)\right)
$$

is either 0 or 1 for any $j$ and $x$.

However, $a_{2}$ and $\alpha_{2}^{1}(x)$ are not logically independent. If you tossed the die, then the initial velocity is surely not zero and the die must have been located somewhere around the table. That is the measurement setting partly specifies the initial conditions. This logical dependence between the element of reality and the measurement setting is called contextuality.

How contextuality leads to the failure of no-conspiracy? First, consider an initial condition $x$ which can reasonably be regarded as "tossing the dice" (that is for the tossing of the die with $x$, it will land on the table and after a couple of rolls it will stop on the table, etc.). For such an $x, \alpha_{2}^{1}(x)$ is algebraically contained in $a_{2}$, therefore

$$
p\left(a_{2} \wedge \alpha_{2}^{1}(x)\right)=p\left(\alpha_{2}^{1}(x)\right) \neq p\left(a_{2}\right) p\left(\alpha_{2}^{1}(x)\right)
$$

if $p\left(a_{2}\right) \neq 1$ and hence no-conspiracy is violated. Second, suppose that $x$ does not count as "tossing the dice" (the die flies over the table, say). Then $a_{2}$ and $\alpha_{2}^{1}(x)$ are algebraically disjoint and hence

$$
p\left(a_{2} \wedge \alpha_{2}^{1}(x)\right)=0 \neq p\left(a_{2}\right) p\left(\alpha_{2}^{1}(x)\right)
$$

if $p\left(a_{2}\right) \neq 0$ and no-conspiracy is again violated. In short, the logical dependence between the measurement settings and the elements of reality directly implies (for non-extremal probabilities, that is typically) a probabilistic dependence between them; that is a violation of no-conspiracy. 
To sum up, even if the elements of reality and the measurement settings are causally detached, they can still violate no-conspiracy if the measurement settings wholly or partially contribute to the definition of the elements of reality. Such a situation cannot be excluded a priori; at least the definitions of the property and the propensity do not exclude it. The logical dependence between elements of reality and measurement settings suffices to establish conspiracy. Contextuality is the other main source for the violation of no-conspiracy.

\section{Discussion}

In this paper we have adopted the following empiricist philosophical position. A physical theory was reconstructed as a formal system plus a semantics connecting the formal system to the world. The semantics has to minimally specify what event types inhabit the world. Event types can be of two sorts: measurement event types and elements of reality. Typically we have direct access to the former but not to the latter. There are two measurement event types: measurement settings and measurement outcomes and there are also two types of elements of reality: properties and propensities. The probability of an event type is understood as simply the long-run relative frequency of the token events instantiating the event type in question. In an experiment the token events are the runs of the experiment.

Adopting the above philosophical position we have argued for the following. No-conspiracy is the requirement that elements of reality should be probabilistically independent of the measurement settings. There is no a priori guarantee that no-conspiracy does hold. If it does, probabilistic relations between the measurement event types will mirror probabilistic relations between the elements of reality. This licenses physics to forget about measurement settings and measurement outcomes and to talk directly about elements of reality. The temptation to delete measurement event types from the semantics of the theory, however, should be resisted.

No-conspiracy is a concept situated within a web of related concepts such as separability, compatibility, causality, locality and contextuality. In the paper I concentrated only on those threads of the web which connected these notions to no-conspiracy. But certainly there are many other interconnections. Causality and contextuality are complementary terms: the more the measurement settings and elements of reality are logically depend on one another, the less room there is for causal connection between them. Separability and spacetime localization do not orient us about causal connections between measurement settings and elements of reality; whereas incompatibility is often due to a direct causal link between them; as in case of the soldiers' shooting and drinking.

Going back to no-conspiracy, the following can be said. Three of the five concepts, namely separability, compatibility and locality do not bring us closer to no-conspiracy. Separability is a weaker concept than no-conspiracy, so one cannot back the latter by the former. Compatibility of measurement settings is empty in case of a purist strategy and only a partial motivation in case of the stubborn strategy. Finally, locality cannot be used to support no-conspiracy at all. However, the remaining two concepts, namely causality and contextuality, are closely linked to no-conspiracy. No-conspiracy can be guaranteed if both causal and logical dependence between the measurement settings and the elements of reality can be excluded. In the first case one needs

to ensure that there is no direct or common causal connection between the individual runs of 
the experiment. In the second case that measurement settings should not contribute to the very definition of the elements of reality.

Whether this can be done and hence a non-conspiratorial physical theory can be provided for a given phenomena is a question that can be answered only by a thorough scrutiny of the phenomena in question. Whether any conspiratorial description of a physical scenario can be replaced by a "better" non-conspiratorial one; whether adopting no-conspiracy can be in conflict, as in the EPR-Bell scenario, with other principles such as local causality, Common Cause Principle, etc.- well, these questions cannot be decided at a general metaphysical level. No-conspiracy is neither an analytic nor a transcendental truth; it is an extra constraint on theory construction the success of which can be decided only on a case-by-case basis.

\section{Appendix}

Throughout the paper we used a simple toy model for a physical theory. Here we provide a general mathematical picture of a physical theory.

Let $a_{i}(i=1 \ldots I)$ be the measurement settings in a given theory and let $A_{i}^{j_{i}}\left(j_{i}=1 \ldots J_{i}\right)$ denote the $j$ th outcome of the $i$ th measurement. Suppose furthermore that there is an element of reality $\alpha_{i}^{k_{i}}\left(k_{i}=1 \ldots K_{i}\right)$ (either a property or a propensity) associated to each measurement setting $a_{i}$ such that

$$
p\left(A_{i}^{j_{i}} \mid a_{i} \wedge \alpha_{i}^{k_{i}}\right)=q_{i}^{j_{i} k_{i}}
$$

where $\sum_{j_{i}=1}^{J_{i}} q_{i}^{j_{i} k_{i}}=1$ for any $i=1 \ldots I$ and $k_{i}=1 \ldots K_{i}$. For a given $i \in I$ the element of reality $\alpha_{i}^{k_{i}}$ is a property iff $J_{i}=K_{i}$ and $q_{i}^{j_{j} k_{i}}=\delta_{j_{i} k_{i}}$. Otherwise $\alpha_{i}^{k_{i}}$ is a propensity.

Suppose that the elements of reality are related nicely to the measurement event types not only in case of a single measurement but also in case of a joint measurement. (Note the word "single" does not mean that the other measurements are not performed; it means rather that it is not taken into consideration whether they are performed or not.) Therefore, select $I^{\prime}$ measurement settings out of the possible $I$ and let now the index $i$ run from 1 to $I^{\prime}$. What we require is that for any such selection (among them the no-selection) the following should hold:

$$
p\left(A_{1}^{j_{1}} \wedge \ldots \wedge A_{I^{\prime}}^{j_{I^{\prime}}} \mid a_{1} \wedge \ldots \wedge a_{I^{\prime}} \wedge \alpha_{1}^{k_{1}} \wedge \ldots \wedge \alpha_{I^{\prime}}^{k_{I^{\prime}}}\right)=q_{1}^{j_{1} k_{1}} \times \cdots \times q_{I^{\prime}}^{j_{I^{\prime}} k_{I^{\prime}}}
$$

Now, the elements of reality $\left\{\alpha_{i}^{k_{i}}\right\}$ are said to satisfy no-conspiracy iff

$$
p\left(a_{1} \wedge \ldots \wedge a_{I} \wedge \alpha_{1}^{k_{1}} \wedge \ldots \wedge \alpha_{I}^{k_{I}}\right)=p\left(a_{1} \wedge \ldots \wedge a_{I}\right) p\left(\alpha_{1}^{k_{1}} \wedge \ldots \wedge \alpha_{I}^{k_{I}}\right)
$$

holds together with those "complemented" variants of (25) where one or more event types are substituted by their complements. From no-conspiracy it follows that they also satisfy noconspiracy for all selections, among them

$$
p\left(a_{i} \wedge \alpha_{i}^{k_{i}}\right)=p\left(a_{i}\right) p\left(\alpha_{i}^{k_{i}}\right)
$$

By means of (24) and no-conspiracy (25) one can transform for any selection the probabilistic relations among the measurement event types into probabilistic relations among elements of 
reality as follows:

$$
p\left(A_{1}^{j_{1}} \wedge \ldots \wedge A_{I^{\prime}}^{j_{I^{\prime}}} \mid a_{1} \wedge \ldots \wedge a_{I^{\prime}}\right)=\sum_{k_{1} \ldots k_{I^{\prime}}} q_{1}^{j_{1} k_{1}} \times \cdots \times q_{I^{\prime}}^{j_{I^{\prime}} k_{I^{\prime}}} p\left(\alpha_{1}^{k_{1}} \wedge \ldots \wedge \alpha_{I^{\prime}}^{k_{I^{\prime}}}\right)
$$

Specifically, if all the event types $\left\{\alpha_{i}^{k_{i}}\right\}$ are properties, then (27) reads as

$$
p\left(A_{1}^{j_{1}} \wedge \ldots \wedge A_{I^{\prime}}^{j_{I^{\prime}}} \mid a_{1} \wedge \ldots \wedge a_{I^{\prime}}\right)=p\left(\alpha_{1}^{j_{1}} \wedge \ldots \wedge \alpha_{I^{\prime}}^{j_{I^{\prime}}}\right)
$$

and in the special case of a single measurement as

$$
p\left(A_{i}^{j_{i}} \mid a_{i}\right)=p\left(\alpha_{i}^{j_{i}}\right)
$$

for all $i=1 \ldots I$. Equation (27) shows that the probability of the outcomes conditioned on the measurement settings is mirrored in the probability of the properties.

Acknowledgements. I wish to thank the members of the Budapest-Kraków Research Group on Probability, Causality and Determinism, especially Márton Gömöri and Balázs Gyenis for valuable discussions. This work has been supported by the Hungarian Scientific Research Fund, OTKA K-115593 and by the Bilateral Mobility Grant of the Hungarian and Polish Academies of Sciences, NM-104/2014.

\section{References}

J. S. Bell, "Free variables and local causality," Epistemological Letters, Feb. (1977).

J. S. Bell, "Bertlmann's socks and the nature of reality," Journal de Physique, Colloque C2, 42, 41-61. (1981).

N. Bohr, "On the Notions of Causality and Complementarity," Dialectica, 2, 312-319 (1948).

N. Bohr, "Discussions with Einstein on epistemological problems in atomic physics," In: Schilpp, P. A. (Ed.), Albert Einstein: Philosopher-Scientist. Vol. 7. Library of Living Philosophers, Evanston, Illinois, 201-241 (1949).

P. W. Bridgman, The Logic of Modern Physics, (New York: The Macmillan Company, 1958).

J. Butterfield, "Vacuum correlations and outcome independence in algebraic quantum field theory" in D. Greenberger and A. Zeilinger (eds.), Fundamental Problems in Quantum Theory, Annals of the New York Academy of Sciences, Proceedings of a conference in honour of John Wheeler, 768-785 (1995).

K. Camilleri and M. Schlosshauer, "Niels Bohr as philosopher of experiment: Does decoherence theory challenge Bohr's doctrine of classical concepts?," Stud. Hist. Phil. Sci., 49(1), 73-83, (2015).

J. Conway and S. Kochen, "The free will theorem," Found. Phys., 36, 1441-1473 (2006).

L. E. Szabó, "The Einstein-Podolsky-Rosen argument and the Bell inequalities," Internet Encyclopedia of Philosophy, URL= http://www.iep.utm.edu/epr/ (2008).

L. E. Szabó, "Mathematics in a physical world," in M. Stannet et al. (eds.), Pre-proceedings of the 3rd Intentional Hypercomputation Workshop (HyperNet 11), TUCS Lecture Notes 14, Turku (2011).

M. Gömöri and G. Hofer-Szabó, "On the meaning of EPR's Criterion of Reality," (in preparation). 
A. Hájek and J. Bub, "EPR," Found. Phys., 22, 313-331 (1992).

H. Halvorson and R. Clifton, "Reconsidering Bohr's reply to EPR," in J. Butterfield and T. Placek (eds). Non-Locality and Modality, Kluwer Academic Publishers, 3-18 (2002).

G. Hofer-Szabó, M. Rédei, and L. E. Szabó, "On Reichenbach's common cause principle and on Reichenbach's notion of common cause," Brit. J. Phil. Sci., 50, 377-399 (1999).

G. Hofer-Szabó, M. Rédei and L. E. Szabó, The Principle of the Common Cause, (Cambridge: Cambridge University Press, 2013).

D. Howard, "What makes a classical concept classical? Toward a reconstruction of Niels Bohr's philosophy of physics," in: Niels Bohr and Contemporary Philosophy. Vol. 158 of Boston Studies in the Philosophy of Science. Kluwer, Dordrecht, pp. 201-229. (1994).

T. Maudlin, "What Bell did," J. Phys. A: Math. Theor., 47, 424010 (2014).

T. Placek and L. Wroński, "On infinite EPR-like correlations," Synthese, 167 (1), 1-32 (2009).

H. Price, Time's Arrow and Archimedes' Point, (Oxford University Press, 1996)

H. Price and K. Wharton, "Disentangling the quantum world," Entropy, 17, (2015).

E. Schrödinger, "The present situation in quantum mechanics," in: J. A. Wheeler and W. H. Zurek, Quantum Theory and Measurement (Princeton Series in Physics vol 23) (Princeton, NJ: Princeton University Press) (Engl. transl. of "Die gegenwärtige Situation in der Quantenmechanik," Naturswissenschaften, 23, 807-49) (1935).

I. Sanpedro, "On Free Will and No-Conspiracy," in: T. Sauer, A. Wüthrich (eds.), New Vistas on Old Problems. Recent Approaches to the Foundations of Quantum Mechanics, (Edition Open Access, 2013).

I. Sanpedro, "Measurement independence, parameter independence and non-locality," Euro Jnl. Phil. Sci., 4, 369-374 (2014).

R. Tumulka, "Comment on 'The free will theorem'," Found. Phys., 37, 186-197 (2007).

B. C. van Fraassen, "The Charybdis of realism: Epistemological implications of Bell's inequality," Synthese, 52, 25-38.

H. Zinkernagel, "Are we living in a quantum world? Bohr and quantum fundamentalism," One hundred years of the Bohr atom: Proceedings from a conference (Edited by F. Aaserud and H. Kragh). Scientia Danica. Series M: Mathematica et physica, vol. 1., 2015. 